\title{
Impact of COVID-19 Pandemic on Haemodialysis Care at a Tertiary Centre in South East Nigeria
}

\author{
Olaronke F. Afolabi1,2*, Monday U. Nwobodo', Chukwuemeka O. Eze', Ugochukwu U. Nnadozie ${ }^{3}$ \\ ${ }^{1}$ Department of Internal Medicine, Alex-Ekweueme Federal University Teaching Hospital, Abakaliki, Nigeria \\ ${ }^{2}$ African Institute for Health Policy and Health Systems, Ebonyi State University, Abakaliki, Nigeria \\ ${ }^{3}$ Division of Plastic Surgery, Department of Surgery, Alex-Ekweueme Federal University Teaching Hospital, Abakaliki, Nigeria \\ Email: *olaronke.afolabi@yahoo.com
}

How to cite this paper: Afolabi, O.F. Nwobodo, M.U., Eze, C.O. and Nnadozie, U.U. (2020) Impact of COVID-19 Pandemic on Haemodialysis Care at a Tertiary Centre in South East Nigeria. Open Journal of Nephrology, 10, 383-387.

https://doi.org/10.4236/ojneph.2020.104038

Received: October 23, 2020

Accepted: December 15, 2020

Published: December 18, 2020

Copyright $\odot 2020$ by author(s) and Scientific Research Publishing Inc. This work is licensed under the Creative Commons Attribution International License (CC BY 4.0).

http://creativecommons.org/licenses/by/4.0/

\begin{abstract}
COVID-19 pandemic has had a significant impact on health services for patients suffering from chronic diseases. Patients suffering from kidney failure require heamodialysis and this modality of treatment is mostly offered in the hospital. Impacts of COVID-19 pandemic on haemodialysis care at a tertiary institution in South East, Nigeria include a reduction in the number of haemodialysis cases during the period of lockdown, an increase in the cost of heamodialysis, scarcity of materials used for the procedure and minor disruption of haemodialylsis care. An improvement in infection control practice at the dialysis unit is observed as a positive effect. It is advocated that the government should assist patients with kidney disease and make the procedure free to ameliorate the health and socio-economic hardships faced by the patients during the pandemic.
\end{abstract}

\section{Keywords}

COVID-19, Heamodialysis Care, Nigeria

\section{Introduction}

Nigeria recorded her first case of COVID-19 on 27th February, 2020 and COVID-19 was declared a pandemic by World Health Organization in March 2020 [1]. With the pandemic, there has been enormous health and socioeconomic implications across the globe.

COVID-19 pandemic has been found to have significant impacts on health services for non-communicable diseases. During the pandemic, patients suffering from chronic diseases have had treatment services disrupted and this is more severe in low and middles income countries. Partial or complete health services 
disruption for hypertension treatment, treatment of chronic diseases and its related complications, cancer treatment and cardiovascular emergencies was reported by world health organization (WHO) [2].

Haemodialysis is a form of renal replacement therapy offered to patients with end stage renal disease or severe forms of acute kidney injury. In Nigeria, heamodialysis is the commonest form of renal replacement therapy [3]. This modality of treatment is not readily available to patients at home in low/middle income countries hence patients usually receive haemodialysis care in the hospital.

This paper describes certain impacts the COVID-19 pandemic has had on haemodialysis practice at Alex Ekwueme Federal University Teaching Hospital, Ebonyi State, South East Nigeria. Alex-Ekwueme Federal Teaching Hospital is a federal tertiary health facility located in Abakaliki, the capital city of Ebonyi state and provides medical services to the people of the state and beyond. While the pandemic has had certain negative impacts, some positive effects may be associated with the pandemic.

\section{Discussion}

\subsection{Reduction in the Number of Haemodialysis Cases during the Pandemic}

The number of patients who accessed haemodialysis care reduced significantly during the lockdown period. From the onset of the pandemic in February, 57 cases were dialysed while 49 cases were dialysed in March. There was a remarkable drop in the number of dialysis done in the months of April (32 cases) and May (27 cases). The number of dialysis cases gradually appreciated in the months of June (39 cases), July (50 cases) and August (48 cases) [Figure 1]. The remarkable reduction in the number of patients coming for haemodialysis observed during this pandemic in the months of March, April and May could be

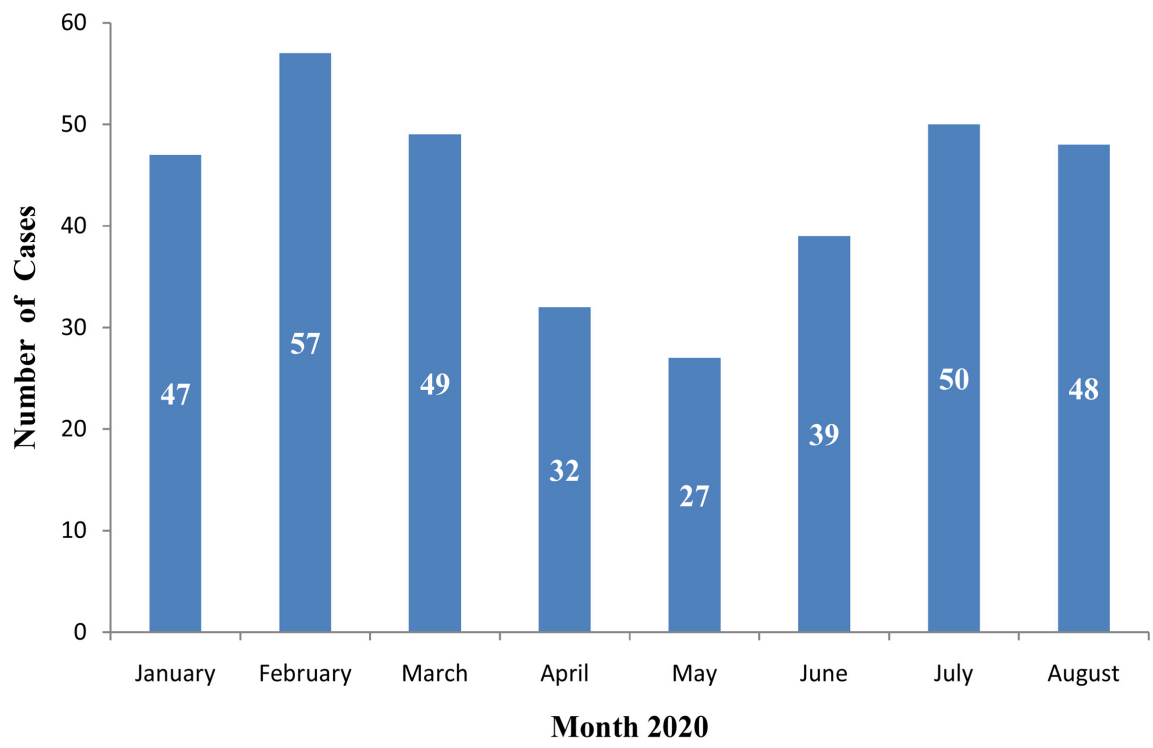

Figure 1. Number of haemodialysis cases during the pandemic. 
due to many reasons. The pandemic scare limited patients' demand for health services. Patients suffering from chronic illnesses which include kidney failure are at risk of developing severe manifestations of COVID-19. Some of these patients feel reluctant accessing health services except on emergency basis because of the fear of contracting the infection in the hospital. Reduction in outpatient services during this pandemic has been observed. WHO reported that $53 \%$ of the countries surveyed have partially or completely disrupted services for hypertension treatment; $49 \%$ for treatment for diabetes and diabetes-related complications; $42 \%$ for cancer treatment, and $31 \%$ for cardiovascular emergencies during the pandemic [2].

Disruption of transport system due to lockdown activities to curtail the spread of the virus could have contributed to the significant reduction in the number of dialysis cases observed in the months of March, April and May. Although the government permitted those on essential services or those who needed essential services to move freely, some of the patients especially those who were coming from neighboring states found it cumbersome coming to the hospital. Cost of transportation also escalated during the pandemic as commercial drivers had to carry fewer passengers due to the social distancing policy.

\subsection{Scarcity and Increase in the Cost of Consumables for Heamodialysis}

Cost of haemodialysis increased from twenty two thousand naira (65 USD) to thirty five thousand naira (100 USD) during the pandemic. Cost of heamodialysis has always been recognized as a major challenge in the provision of care for patients with kidney failure in Sub-Sahara Africa [4] [5]. Haemodialysis cost increased by about $50 \%$ during this period. This is majorly due to an increase in the cost of materials (consumables) used for the procedure. These materials are not produced locally in the country; hence disruption in both local and international travels during the pandemic significantly increased the cost of these materials. Disruption in both local and international travels during the pandemic also accounted for scarcity of these products during this period such that some patients experienced a delay or cancellation of dialysis procedure during the pandemic. This caused minor disruption in service delivery observed in the dialysis unit during the COVID-19 pandemic. Major reasons for discontinuing services for non-communicable diseases reported by WHO during the pandemic included shortage of medicines, diagnostics and other technologies [2].

COVID-19 pandemic has had far reaching effects on the populace with consequent socio-economic implications. Many people have lost their jobs and suffered serious economic/financial difficulties. An increase in the cost of haemodialysis at such period definitely aggravated the financial difficulties faced by patients and their relatives. The modest increase in the cost of haemodialysis has great implications since almost all the patients pay out of pocket for heamodialysis. Ngbabe et al. reported that in Congo, heamodialysis is made free to all patients during the pandemic [6]. The initiative is highly commendable. 


\subsection{Improved Infection Control Practice}

The pandemic has had positive effect on infection control practice. During the pandemic, strict infection control protocol is put in place in order to curtail the spread of the virus. Hand hygiene (hand washing and hand sanitization) is instituted at the hospital gate (before entering the hospital), at the entry of the dialysis unit, before attending to a patient, after attending to each patients/in between patients and before aseptic procedures. Also, the use of facemasks is mandatory to all patients, patients' relatives and medical personnel in the dialysis unit. While patients and their relatives could use either a cloth mask or a surgical mask, medical personnel are provided with surgical masks for protection.

Furthermore, temperature of patients is usually checked on arrival at the dialysis unit and patients with temperature higher than $37.5^{\circ} \mathrm{C}$ are further evaluated. In addition, patients are also screened for COVID-19 specific symptoms like cough, breathlessness, loss of smell and loss of taste. Regular cleaning and disinfection of the dialysis unit, the waiting area, surfaces and equipment including dialysis chairs, counter tops and external parts of the dialysis machines is also done.

Infection control practice has improved considerably during the pandemic. Though the pandemic is gradually easing off, it is expected that this positive effect will be long lasting such that health personnel and patients will continue these preventive measures as routine practices after the pandemic.

During this pandemic, none of our haemodialysis patients has been confirmed positive for COVID-19. It is commendable that during the pandemic, the hospital has continued to offer haemodialysis care to patients despite the challenges.

\subsection{Health Policy Implications}

It is clear that heamodialysis cost is unbearable for our patients with kidney failure because majority of our patients pay out of pocket [3] [4]. Expenditures for such life saving procedures should be made free especially during a pandemic like this.

COVID-19 pandemic has the potential to increase poverty and health inequalities especially in low income countries. It is advocated that the federal government should assist patients by making haemodialysis procedure free as reported in some other African countries like Congo [6] during this pandemic to reduce the health and socioeconomic burdens of the pandemic on patients with kidney failure. Inclusion of heamodialysis care of patients in the COVID-19 palliative measures offered by the federal government will be of huge benefit to the patients.

\section{Conclusions and Recommendations}

COVID-19 pandemic has had remarkable implications on haemodialysis care. Reduction in the number of dialysis cases, substantial increase in the cost of heamodialysis, scarcity of haemodialysis materials and minor disruptions in 
haemodialysis care are observed during this pandemic; however the pandemic has led to improvement of infection control practice.

Strict infection control practice should be observed in all haemodialysis units to minimize the spread of the virus. Also, the government should ease the financial burden of patients on renal replacement therapy because most of them cannot bear the cost; hence there must be advocacy at all levels for this to be actualized.

\section{Conflicts of Interest}

The authors declare no competing interests.

\section{References}

[1] Paintsil, E. (2020) COVID-19 Threatens Health Systems in Sub-Saharan African: The Eye of the Crocodile. The Journal of Clinical Investigation, 130, 2741-2744. https://doi.org/10.1172/JCI138493

[2] WHO (2020) COVID-19 Significantly Impacts Health Services for Non Communicable Diseases. News Release.

[3] Dada, S.A., Ajite, A.B., Ibitoba, F.A., Thomas, A.A., Dada, O.E., et al. (2019) Challenges of Haemodialysis: A Single Centre Experience in South West Nigeria. Journal of Clinical Nephrology, 3, 55-60. https://doi.org/10.29328/journal.jcn.1001026

[4] Naicker, S. (2016) Nephrology in Africa-Challenges of Practice in Resource-Limited Environment. Clinical Nephrology, 86, 84-89. https://doi.org/10.5414/CNP86S112

[5] Ijoma, C.K., Ulasi, I.I. and Kalu, A.O. (1998) Cost Implications of Treatment of End-Stage Renal Disease in Nigeria. International Journal of Medicine and Health Development, 3, 95-96.

[6] Ngabe, P., Yattara, H., Baoua, D., Loumingou, R. and Moukassa, D. (2020) Managing a Hemodialysis Unit in Times of Pandemic to COVID-19. Experience of Edith Lucie Bongo Ondimba General Hospital. Open Journal of Nephrology, 10, 223-226. https://doi.org/10.4236/ojneph.2020.103021 